\title{
A simple test for the ignorability of non-compliance in experiments
}

\section{Martin Huber}

March 29, 2013

University of St. Gallen, Dept. of Economics

\begin{abstract}
This papers proposes a simple method for testing whether non-compliance in experiments is ignorable, i.e., not jointly related to the treatment and the outcome. The approach consists of (i) regressing the outcome variable on a constant, the treatment, the assignment indicator, and the treatment/assignment interaction and (ii) testing whether the coefficients on the latter two variables are jointly equal to zero. A brief simulation study illustrates the finite sample properties of the test.
\end{abstract}

Keywords: experiment, treatment effects, non-compliance, endogeneity, test.

JEL classification: $\mathrm{C} 12, \mathrm{C} 21, \mathrm{C} 26$.

Address for correspondence: Martin Huber, SEW, University of St. Gallen, Varnbüelstrasse 14, 9000 St. Gallen, Switzerland, martin.huber@unisg.ch. 


\section{Introduction}

Non-compliance in experiments arises if some units receive a treatment different to the random assignment. While the average treatment effect (ATE) on the subgroup of compliers (whose treatment complies with the assignment) is identified under some conditions, one typically prefers to make causal statements for the entire experimental population. In general, however, the ATEs on compliers and the entire population are only equivalent if non-compliance is ignorable, implying that compliers and non-compliers are comparable in terms of unobservables affecting the outcome.

Given that the assignment is random, does not directly affect the outcome, and influences the treatment, the ignorability of non-compliance has a testable implication: The assignment must be independent of the outcome conditional on the treatment. Conversely, if non-compliance is non-ignorable and causes treatment confounding, conditioning on the latter generally introduces dependence between the assignment and outcome. We propose testing ignorability by (i) regressing the outcome on a constant, the treatment, the assignment, and the treatment/assignment interaction and (ii) jointly testing for zero coefficients on the latter two variables.

A conceptual extension is testing conditional ignorability given observed covariates, for which de Luna and Johansson (2012) proposed a nonparametric method. Donald, Hsu, and Lieli (2011) suggest a further test requiring one-sided non-compliance (no unit randomized out gets the treatment) under which the ATE on the treated compliers corresponds to that on all treated. This paper focuses on unconditional ignorability, but conditional testing is straightforward if covariates are discrete (by testing within cells of covariate values or based on a fully interacted regression model). Section 2 discusses the model and testing, Section 3 presents a simulation study.

\section{Model and testing}

We are interested in the ATE of a binary treatment $D$ on an outcome $Y$. In experiments, the treatment is randomly assigned. Let $Z$ denote the binary assignment indicator. In the presence of 
non-compliance, $Z \neq D$ for a subgroup of units, implying that $D$ is not a deterministic function of $Z$. We consider the following general models for $Y$ and $D$ :

$$
\begin{aligned}
& Y=g(D, U), \\
& D=I\{h(Z, V) \geq 0\} .
\end{aligned}
$$

$g$ and $h$ denote unknown functions, $U$ and $V$ are unobserved terms in the outcome and treatment equations, and $I\{\cdot\}$ is the indicator function. To give an example, $Z$ may be random assignment to a training program, $D$ actual program participation, $Y$ earnings after assignment, and $V$ and $U$ unobserved measures of motivation to take the treatment and at work, respectively.

To translate our model into the potential outcomes framework, see for instance Rubin (1974), denote by $Y(d)$ the potential outcome when setting the treatment to $d$ and by $D(z)$ the potential treatment when setting the assignment to $z$, with $d, z \in\{0,1\}$. Exogenously fixing $d, z$ in (1) and (2) yields the potential outcomes and treatments:

$$
\begin{aligned}
& Y(d)=g(d, U), \\
& D(z)=I\{h(z, V) \geq 0\} .
\end{aligned}
$$

If $V$ affects $D$ conditional on $Z$, non-compliance exists and $D(z) \neq z$ for some units. Furthermore, if $V$ and $U$ are associated as it appears plausible in our example with unobserved motivation, non-compliance is non-ignorable and $D$ is confounded by $U$.

We now discuss assumptions under which the independence of $V$ and $U$, which implies the ignorability of non-compliance, can be tested if data sampling is i.i.d.

Assumption 1: $Z \perp(U, V)$.

' $\perp$ ' denotes independence. Along with our model, Assumption 1 implies that $Z$ is randomly assigned (by its independence of $V$ ) and does not have a direct effect on $Y$ (by its independence of $U$ ). In terms of potential outcome notation, $Z$ is independent of the joint distribution of 
potential outcomes and treatment states: $Z \perp\{Y(1), Y(0), D(1), D(0)\}$.

Assumption 2: $Z$ and $D$ are not independent.

In experiments, Assumption 2 appears reasonable because assignment should affect the treatment of some units, for which $I\{h(1, V) \geq 0\} \neq I\{h(0, V) \geq 0\}$ or equivalently, $D(1) \neq D(0)$. Those whose treatment reacts to the assignment are either compliers $(D(1)=1$ and $D(0)=0)$ or defiers $(D(1)=0$ and $D(0)=1)$. In contrast, the treatment states of always takers $(D(1)=1$ and $D(0)=1)$ and never takers $(D(1)=0$ and $D(0)=0)$ are not affected by the assignment. To identify the complier ATE, Angrist, Imbens, and Rubin (1996) assume compliers $(\operatorname{Pr}(D(1)>$ $D(0))>0$ ) but rule out defiers $(\operatorname{Pr}(D(1) \geq D(0))=1)$, which is a special case of Assumption 2 .

Our third assumption states that non-compliance is ignorable. This is the restriction to be tested, conditional on the satisfaction of Assumptions 1 and 2.

\section{Assumption 3: $V \perp U$.}

Assumptions 1 and 3 together imply that $D \perp U$ or in terms of potential outcomes, that $D \perp Y(d)$. I.e., the treatment only randomly deviates from the assignment so that the distribution of $U$ (or $Y(d))$ is equal among compliers and in the entire population. If Assumption 2 holds, too, we obtain the following testable implication:

$$
Z \perp Y \mid D
$$

To see this, note that Assumptions 1 and 2 are not sufficient for (5): If $D$ is associated with $Z$ (Assumption 2), then $Z \perp U \mid D$ (or $Z \perp Y \mid D$ ) does not necessarily hold even if $Z \perp Y$ (Assumption 1). Because if $U$ is related to $V$ and thus confounds $D$, conditioning on the latter introduces a dependence between $Z$ and $Y$, even though the true effect of $Z$ on $Y$ is zero due to Assumption 1. However, if we impose Assumption 3, also $D \perp U$ such that $Z \perp U \mid D$, which implies $Z \perp Y \mid D$ by our model. Therefore, Assumption 3 can be tested by verifying (5).

Figure 1 graphically presents our model both with and without Assumption 3, where each arrow represents a causal relation, see for instance Pearl (1995) for a discussion of causal diagrams. 
Figure 1: Directed acyclical graphs under independence (a) and dependence (b-d) of $U, V$
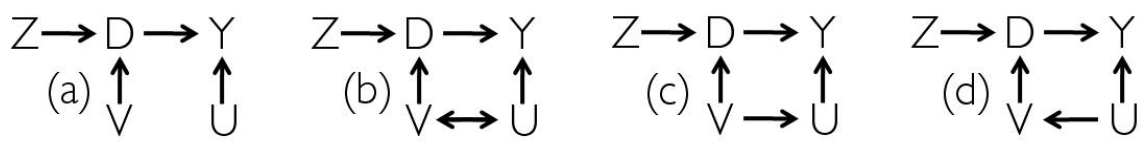

In (a), Assumptions 1 to 3 are satisfied and conditioning on $D$ does not entail dependence between $Z$ and $U$ (or $Y(d)$ ). In (b), (c), and (d), however, Assumption 3 is violated due to dependence between $V$ and $U$. Therefore, conditioning on $D$ introduces an association between $U$ and $Z$ (and thus, $Y(d)$ and $Z)$.

For testing (5), we suggest to (i) regress $Y$ on a constant, $D, Z$, and the interaction $D \cdot Z$ (and to compute robust standard errors if heteroskedasticity should be allowed for) and (ii) jointly test whether the coefficients on $Z$ and $D \cdot Z$ are equal to zero by a Wald or F-test. Using OLS regression, this amounts to testing whether $E(Y \mid D=0, Z=0)=E(Y \mid D=0, Z=1)$ (reflected by the coefficient on $Z$ ) and $E(Y \mid D=1, Z=1)=E(Y \mid D=1, Z=0$ ) (reflected by the sum of coefficients on $Z$ and $D \cdot Z$ ), because the model is fully saturated. This is an unconditional version of de Luna and Johansson (2012), who nonparametrically test for equalities in means given observed covariates. However, note that mean independence is necessary, but not sufficient for (5), postulating full independence between $Z$ and $Y$ given $D$. Instead of OLS, quantile regression may therefore be used to test general distributional features. On the other hand, if one is solely interested in ATEs (rather than quantile treatment effects), mean independence suffices for the innocuousness of non-compliance and we will henceforth focus on this case.

To see why testing verifies the ignorability of non-compliance, let $T \in\{a, c, \bar{d}, n\}$ be the compliance type, where $a$ denotes the always takers, $c$ the compliers, $\bar{d}$ the defiers, and $n$ the never takers. Any $E(Y \mid D=d, Z=z)$ with $d, z \in\{0,1\}$ consists of a mixture of mean potential outcomes of (at most) two types. E.g., an observation with $D=1, Z=1$ implies that $D(1)=1$ and may thus either be a complier or an always taker. However, as the counterfactual treatment under $Z=0$ is unknown, the exact type cannot be determined. Denote by $E(Y(d) \mid T=t)$ the mean potential outcome of type $t$ under treatment $d$ and by $\pi_{t}=\operatorname{Pr}(T=t)$ the share of a 
particular type in the population. Assumption 1 states that $E(Y(d) \mid T=t, Z=z)=E(Y(d) \mid T=$ t) and $\operatorname{Pr}(T=t \mid Z=z)=\pi_{t}$, because the assignment does neither directly affect the outcome nor is it related to the types (or the potential treatments) due to its randomness. Therefore, each observed conditional mean outcome corresponds to a mixture of mean potential outcomes:

$$
\begin{aligned}
& E(Y \mid D=1, Z=1)=\frac{\pi_{a}}{\pi_{a}+\pi_{c}} \cdot E(Y(1) \mid T=a)+\frac{\pi_{c}}{\pi_{a}+\pi_{c}} \cdot E(Y(1) \mid T=c), \\
& E(Y \mid D=1, Z=0)=\frac{\pi_{a}}{\pi_{a}+\pi_{\bar{d}}} \cdot E(Y(1) \mid T=a)+\frac{\pi_{\bar{d}}}{\pi_{a}+\pi_{\bar{d}}} \cdot E(Y(1) \mid T=d), \\
& E(Y \mid D=0, Z=0)=\frac{\pi_{n}}{\pi_{n}+\pi_{c}} \cdot E(Y(0) \mid T=n)+\frac{\pi_{c}}{\pi_{n}+\pi_{c}} \cdot E(Y(0) \mid T=c), \\
& E(Y \mid D=0, Z=1)=\frac{\pi_{n}}{\pi_{n}+\pi_{\bar{d}}} \cdot E(Y(0) \mid T=n)+\frac{\pi_{\bar{d}}}{\pi_{n}+\pi_{\bar{d}}} \cdot E(Y(0) \mid T=d) .
\end{aligned}
$$

Testing $(6)=(7)$ and $(8)=(9)$ has in general non-trivial power to detect heterogeneity in mean potential outcomes across types, which apart from special cases would imply that the ATEs differ across types, too. Then, non-compliance would be non-ignorable, because the ATE of one type (e.g. the compliers) would not be representative for the entire population. As a final remark, note that outcome attrition and non-response (which are not considered here) could compromise the interpretation of the test, e.g. by introducing selection bias that may give power to the test even though non-compliance is ignorable.

\section{Simulation}

Our simulation study is based on the following data generating process:

$$
\begin{aligned}
Y & =D+D \cdot U+U \\
D & =I\{-0.5 \beta+\beta Z+V \geq 0\}, \quad Z \sim \operatorname{Bernoulli}(0.5) \\
\left(\begin{array}{c}
U \\
V
\end{array}\right) & \sim \mathcal{N}(\mu, \sigma), \text { where } \mu=\left(\begin{array}{l}
0 \\
0
\end{array}\right) \text { and } \sigma=\left(\begin{array}{cc}
1 & \lambda \\
\lambda & 1
\end{array}\right) .
\end{aligned}
$$


The effect of $D$ on $Y$ is heterogeneous due to the interaction $D \cdot U$. Therefore, ATEs on the compliers and the entire population are different if these groups differ in terms of $U$. $\beta$ determines the strength of $Z$ on $D$ and is set to 0.5 and 1.5, entailing roughly $20 \%$ and $55 \%$ of compliers, respectively. $\lambda$ represents the covariance between $U$ and $V$ and non-compliance is ignorable only if $\lambda=0$.

Table 1: Simulation results

\begin{tabular}{|c|c|c|c|c|c|c|c|c|c|c|c|c|}
\hline & \multicolumn{6}{|c|}{ covariance $(\lambda)$ of $U, V$ is zero } & \multicolumn{6}{|c|}{ covariance $(\lambda)$ of $U, V$ is 0.5} \\
\hline & \multicolumn{3}{|c|}{$\beta=0.5(\overline{20 \% \text { compl. })}$} & \multicolumn{3}{|c|}{$\beta=1.5$ (55\% compl.) } & \multicolumn{3}{|c|}{$\beta=0.5(\overline{20 \% \text { compl. })}$} & \multicolumn{3}{|c|}{$\beta=1.5$ (55\% compl.) } \\
\hline & $\operatorname{rf} 5 \%$ & $\operatorname{rf} 10 \%$ & $\mathrm{~b}_{\mathrm{ATE}}$ & $\operatorname{rf} 5 \%$ & rf $10 \%$ & $\mathrm{~b}_{\mathrm{ATE}}$ & $\operatorname{rf} 5 \%$ & rf $10 \%$ & $\mathrm{~b}_{\mathrm{ATE}}$ & $\operatorname{rf} 5 \%$ & rf $10 \%$ & $\mathrm{~b}_{\mathrm{ATE}}$ \\
\hline$N$ & 0.050 & 0.092 & 0.007 & 0.065 & 0.116 & 0.003 & 0.115 & 0.196 & 1.157 & 0.477 & 0.592 & 0.908 \\
\hline$N=400$ & 0.062 & 0.108 & -0.002 & 0.050 & 0.096 & 0.001 & 0.278 & 0.408 & 1.154 & 0.970 & 0.990 & 0.904 \\
\hline$N=1600$ & 0.053 & 0.100 & 0.001 & 0.063 & 0.101 & 0.000 & 0.875 & 0.917 & 1.161 & 1.000 & 1.000 & 0.904 \\
\hline
\end{tabular}

Note: rf $5 \%, 10 \%$ : Rejection frequencies at the $5 \%$ and $10 \%$ significance level. $\mathrm{b}_{\mathrm{ATE}}$ : Bias of the ATE.

Table 1 reports the rejection frequencies of the Wald test (with heteroskedasticity robust covariance matrix) at the $5 \%$ and $10 \%$ significance levels (rf 5\%, rf 10\%) for 1000 simulations and three sample sizes $N$. The bias of estimating the ATE on the entire population by the mean difference in treated and non-treated outcomes is also provided $\left(\mathrm{b}_{\mathrm{ATE}}\right)$. For $\lambda=0$, the rejection frequencies are close to the nominal sizes while estimation bias is small and decreasing in $N$. For $\lambda=0.5$ (violation of Assumption 3), the bias is substantial and does not vanish asymptotically. The test gains power in the share of compliers and $N$. Our results suggest that testing is powerful even in moderate samples if the complier population is not too small.

\section{References}

Angrist, J., G. Imbens, and D. Rubin (1996): "Identification of Causal Effects using Instrumental Variables," Journal of American Statistical Association, 91, 444-472 (with discussion).

De Luna, X., And P. Johansson (2012): "Testing for Nonparametric Identification of Causal Effects in the Presence of a Quasi-Instrument," IZA Discussion Paper No. 6692.

Donald, S. G., Y.-C. Hsu, and R. P. Lieli (2011): "Testing the Unconfoundedness Assumption via Inverse Probability Weighted Estimators of (L)ATT," unpublished mansucript, University of Texas, Austin.

Pearl, J. (1995): "Causal Diagrams for Empirical Research," Biometrika, 82, 669-688.

Rubin, D. B. (1974): "Estimating Causal Effects of Treatments in Randomized and Nonrandomized Studies," Journal of Educational Psychology, 66, 688-701. 